\title{
Salvage high-dose-rate brachytherapy for prostate cancer persistence after brachytherapy: repeated use of a polyethylene glycol hydrogel spacer
}

\author{
Rodrigo Hepp, MDl, Thilo Eggert, MD², Georg Schabl, MSc', Lars Herberholz, MSc', Tim Petry, cand. med. ${ }^{3}$, \\ Razvan Galalae, MD1.3 \\ IKlinik für Strahlentherapie und Onkologie, Evangelische Kliniken Gelsenkirchen, ${ }^{2}$ Urologische Praxis, Urologie, Haltern am See, ${ }^{3}$ Medizinische \\ Fakultät, Christian-Albrechts-Universität zu Kiel, Germany
}

\begin{abstract}
Purpose: The aim of this study is to determine if a repeated hydrogel injection in a previously irradiated patient prior to salvage high-dose-rate brachytherapy (HDR-BT) is feasible.

Material and methods: A 61-year-old man with an organ confined (cT1c cN0 cM0, Gleason score $3+3=6$, initial prostate-specific antigen [PSA] $7.9 \mathrm{ng} / \mathrm{ml}$ ) prostate cancer was previously treated with HDR-BT (3 fractions of 11.5 Gy every $2^{\text {nd }}$ week) after hydrogel injection to reduce the rectal dose. Ten months after, an isolated local persistence was seen on a PSMA PET-CT. Nadir PSA was $2.0 \mathrm{ng} / \mathrm{ml}, 3$ months after treatment and was $3.95 \mathrm{ng} / \mathrm{ml}$ by the re-treatment. Salvage therapy consisted of HDR-BT ( 3 fractions of 9 Gy every $2^{\text {nd }}$ week) with a simultaneous integrated boost to the residual region. Again, a hydrogel injection $(10 \mathrm{ml})$ was applied to reduce the rectal dose prior to the treatment.

Results: Both hydrogel injection and salvage HDR-BT could be applied without any significant complications or toxicity. A good PSA response was observed with a nadir of $0.42 \mathrm{ng} / \mathrm{ml}$, twelve months after salvage therapy. Acute toxicity (max grade II) resolved within 2 days after treatment.

Conclusions: The use of a hydrogel prior to salvage HDR-BT in a patient previously treated with HDR-BT is feasible and could help reduce the rectal exposure in the salvage setting.

Key words: brachytherapy, hydrogel, prostate cancer, rectal spacer, salvage.

\section{Introduciotn}

The patient is a fit (ECOG performance status 0) and active 61-year-old man, who has been initially diagnosed outside of our institution with a prostate cancer in December 2014. Thirty months before diagnosis, his routine prostate-specific antigen (PSA) was $3.41 \mathrm{ng} / \mathrm{ml}$ but rose to $7.94 \mathrm{ng} / \mathrm{ml}$ in November 2014. Digital rectal examination was negative. A transrectal ultrasound-guided biopsy revealed a Gleason score $6(3+3)$ adenocarcinoma in $5 \%$ of $2 / 10$ (left apex and right base) cores. No magnetic resonance imaging (MRI) was performed. An ${ }^{18} \mathrm{~F}$-choline positron emission tomography/computed tomography (PET/CT) showed an avid but inhomogeneous tracer uptake in the left and middle prostate lobes with a maximum standardized uptake value $\left(\mathrm{SUV}_{\max }\right)$ of 5.8 . No nodal involvement or distant metastases were documented. The patient was classified as per the American Joint Committee on Cancer (AJCC) $7^{\text {th }}$ Edition stage I (cT1c
cN0 cM0) and taken into an active-surveillance program, but in July 2015, the PSA rose to $10.77 \mathrm{ng} / \mathrm{ml}$ (AJCC stage IIA) and the patient was referred to our clinic for treatment.

\section{Initial therapeutic plan}

After a multidisciplinary consultation, including urologists, radiation and medical oncologists and radiologists, the patient was selected to proceed with a high-dose-rate (HDR) fractionated prostate brachytherapy (BT) as single treatment modality to a total dose of $34.5 \mathrm{~Gy}$ in 3 fractions, every $2^{\text {nd }}$ week. After written informed consent, the patient was also enrolled in a prospective surveillance clinical trial (NCT01999660) to evaluate the use of a polyethylene glycol hydrogel spacer (Space-OAR; Augmenix Inc., Waltham, MA, USA) to reduce the rectal exposure in prostate radiotherapy patients. 


\section{Hydrogel injection}

The hydrogel application technique has been published elsewhere [1,2]. Briefly, under general anesthesia and with the patient in lithotomy position, ten milliliters of hydrogel spacer were injected in the perirectal space. The application runs under transrectal ultrasound (TRUS) guidance using an $18 \mathrm{G}$ transperineal needle. The space between Denovilliers' fascia and the anterior rectal wall was dissected using $10 \mathrm{ml}$ of injectable saline solution just before injecting the spacer. The first HDR-BT implant was planned 12 days after hydrogel injection to allow the hydrogel to stabilize and resorption of possible air injection.

\section{High-dose-rate brachytherapy procedure}

Following the Kiel University philosophy of HDR-BT planning [3], we optimized our 3D dose distributions to intentionally overdose the peripheral zone of the prostate using a two-step optimization: hybrid inverse treatment planning and optimization (HIPO) for the pre-plan, and a graphical optimization (GO) for the live plan.

The HDR-BT was performed under general anesthesia every second week. Treatment plans were computed using Oncentra Prostate (Nucletron, an Elekta company, Elekta AB, Stockholm, Sweden). Under TRUS guidance, the prostate (CTV), urethra, bladder, and rectum were contoured. Also, the peripheral zone (CTV2) was outlined. A HIPO for needle placement geometry was used. Catheters ( 15 for the first two and 16 for the last implant) were placed transperineally using a dedicated template. After checking the position of all needles at the reference plane, each catheter was pushed to its final position, about $1 \mathrm{~cm}$ above the prostate base, using live ultrasound longitudinal image guidance. After reconstructing the actual position of the catheters and correcting any displacement of the contoured volumes, a new HIPO for the dwell-times was run. Finally, a GO if necessary, was also performed. Then, the treatment was applied using a microSelectron treatment delivery system (Nucletron, an Elekta company, Elekta AB, Stockholm, Sweden). Typically, a brachytherapy implant procedure took less than two hours.

\section{Initial gel injection result and dosimetry of the implants}

Distances between rectal wall and prostate capsule at the base, apex, and mid-plane of the prostate before gel injection and again before the first brachytherapy implant (Table 1) were measured. Three implants were planned

Table 1. Distance between prostate and rectum before and after hydrogel injection. Initial treatment

\begin{tabular}{lccc} 
& Before & After & Delta \\
\hline Base $(\mathrm{mm})$ & 13.1 & 16.4 & 3.3 \\
\hline Apex $(\mathrm{mm})$ & 6.6 & 14.0 & 7.4 \\
\hline Reference $(\mathrm{mm})$ & 4.7 & 12.4 & 7.7
\end{tabular}

to deliver 11.5 Gy to $D_{90}$ (dose delivered to $90 \%$ of the CTV) each. Other CTV constraints were $V_{100}$ (volume of CTV receiving $100 \%$ of the dose) $\geq 90 \%$ and $\mathrm{V}_{150} \leq 50 \%$. For the peripheral zone (CTV2), $\mathrm{D}_{90}$ should be $\geq 120 \%$. The constraints for organs at risk (OAR) were: urethra, $\mathrm{D}_{0.1 \mathrm{cc}}$ (maximal dose applied to a volume of $0.1 \mathrm{~cm}^{3}$ ) $\leq 130 \%$, and $\mathrm{D}_{2 \mathrm{cc}}$ for rectum and bladder $\leq 75 \%$ of the prescribed dose. As shown in Table 2, all three implants met the given dose constraints, but also with an impressive sparing of the rectum $\left(D_{2 c c}<55 \%\right)$ in all implants.

\section{Acute toxicity and prostate-specific antigen values during initial follow-up}

The patient was controlled regularly every 3 months, including serum PSA determination. Acute toxicity according to the common toxicity criteria for adverse events (CTAAE) version 4.3 was mild (grade I dysuria) and disappeared 3 weeks after the end of treatment. There was no gastrointestinal toxicity reported. The PSA value decreased to a nadir of $2.0 \mathrm{ng} / \mathrm{ml}$ three months after the last implant, but thereafter, it began to rise again and 10 months after treatment, it reached $3.95 \mathrm{ng} / \mathrm{ml}$. Eleven months after the initial treatment, a PSMA PET/CT was performed showing a local residual disease on the left base of the prostate with extension to the seminal vesicle base ipsilateral (Figure 1). No regional or distant metastases were detected.

\section{Salvage therapy}

After discussing different therapeutic alternatives with the patient, including surgery and external beam radiotherapy, the patient opted for a HDR-BT re-irradiation as a salvage therapy. Three implants two weeks apart with an intended dose of 9 Gy (prescribed to $D_{90}$, with a $V_{100} \geq 85 \%$ ) and a simultaneous integrated boost (intended dose $\geq 115 \%$ of the $\mathrm{D}_{90}$ for the prostate) to the PET $(+)$ regions were planned. Once more, in order to reduce as far as possible the rectal dose, a second gel injection was performed a week before the first brachytherapy salvage implant (Figure 2). The technique used for the gel injection and brachytherapy application did not differ from the ones used during the initial treatment.

Table 2. Dosimetric parameters of the initial brachytherapy implants

\begin{tabular}{lccc} 
& 1. Implant & 2. Implant & 3. Implant \\
\hline Prostate $\mathrm{D}_{90}(\mathrm{~Gy})$ & 11.7 & 12.2 & 11.6 \\
\hline Prostate $\mathrm{V}_{100}(\%)$ & 90.8 & 92.5 & 90.3 \\
\hline Boost $\mathrm{D}_{90}(\mathrm{~Gy})$ & 14.7 & 14.5 & 14.2 \\
\hline Rectum $\mathrm{D}_{2 \mathrm{~cm}}(\%)$ & 53.7 & 54.3 & 42.3 \\
\hline Bladder $\mathrm{D}_{2 \mathrm{~cm}^{3}}(\%)$ & 74.3 & 68.0 & 63.9 \\
\hline Urethra $\mathrm{D}_{0.1 \mathrm{~cm}^{3}}(\%)$ & 128 & 133 & 128
\end{tabular}

$D_{90}$ - dose delivered to $90 \%$ of the CTV, $V_{100}$-volume of CTV receiving $100 \%$ of the dose, $D_{2 \mathrm{~cm}^{3}}$ - maximal dose applied to a volume of $2 \mathrm{~cm}^{3}, D_{0.1 \mathrm{~cm}^{3}}$ - maximal dose applied to a volume of $2 \mathrm{~cm}^{3}$ 


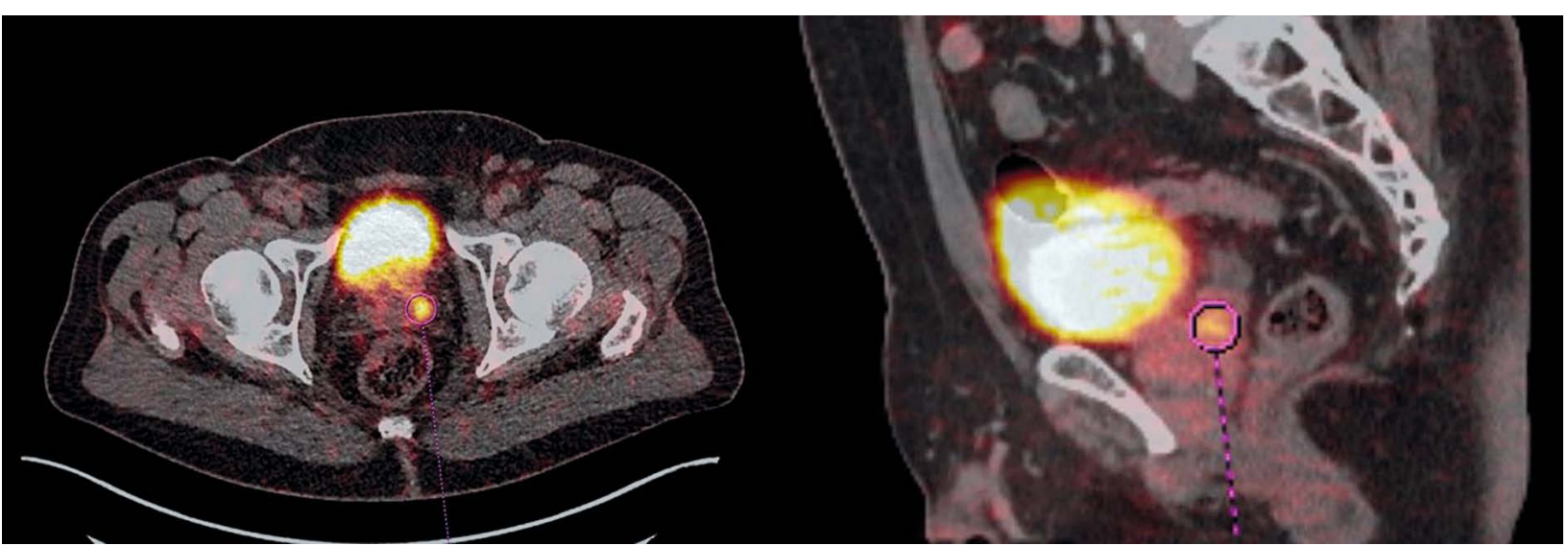

Fig. 1. PSMA PET/CT showing residual region

\section{Gel injection result and dosimetry of the salvage implants}

Again, the distance between rectal wall and prostate capsule at the base, apex, and mid-plane of the prostate before and after gel injection were measured (Table 3). During brachytherapy dose optimization, a higher value was applied to protect the urethra accepting a modest unsubstantial underdosage $\left(\mathrm{V}_{100} \geq 85 \%\right)$ around it but giving a $10 \%$ overdose to the PET $(+)$ regions. The dosimetric parameters of the salvage implants are shown in Table 4.

\section{Acute toxicity and PSA values after salvage therapy}

The acute toxicity was repeatedly mild and limited to dysuria/mictional discomfort grade II in the first two days after the implants, and resolved completely a week after the end of treatment.

By the last follow-up visit (12 months after salvage treatment), the patient was fully asymptomatic with a PSA of $0.42 \mathrm{ng} / \mathrm{ml}$.

\section{Discussion}

Patients with an organ confined relapse after initial radiation treatment, represents a challenging clinical scenario. As in this situation, a reasonably curative potential exists and in the absence of randomized clinical evidence, a careful evaluation in a multidisciplinary setting of treatment options and possible complications and late sequelae is mandatory. The National Comprehensive Cancer Network (NCCN) guidelines [4] consider possible candidates for curative intended treatment in a relapse

Table 3. Distance between prostate and rectum before and after hydrogel injection. Salvage treatment

\begin{tabular}{lccc} 
& Before & After & Delta \\
\hline Base $(\mathrm{mm})$ & 4.3 & 7.2 & 2.9 \\
\hline Apex $(\mathrm{mm})$ & 6.0 & 16.6 & 10.6 \\
\hline Reference $(\mathrm{mm})$ & 4.9 & 10.2 & 5.3
\end{tabular}

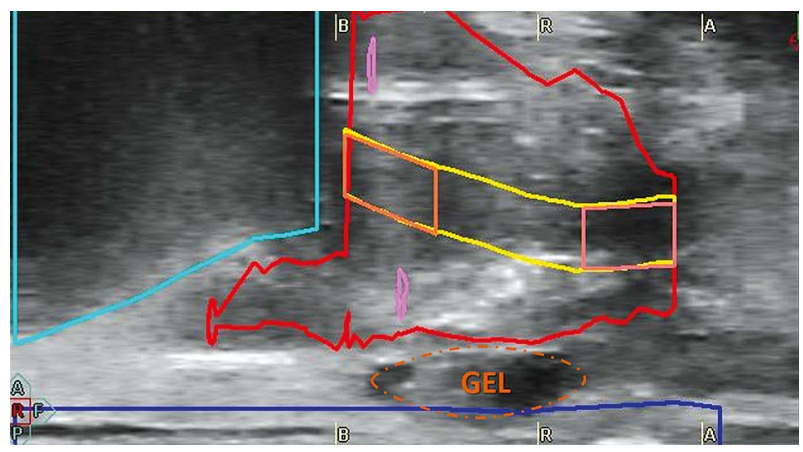

Fig. 2. Ultrasound image showing the hydrogel during the brachytherapy procedure

setting patients with a T1-T2 tumor, PSA by the time of recurrence $<10 \mathrm{ng} / \mathrm{ml}$, and life expectancy $>10$ years. Treatment alternatives include surgery, cryoablation, or reirradiation $[5,6,7,8]$. The choice of a particular treatment option relies typically on patients' preferences and the treatment center experience with a specifically modality expertise.

In this context, HDR-BT emerges as a valuable alternative with high effectivity and low morbidity. In a recent published review, Chatzikonstantinou et al. [8] summarized the published evidence of HDR-BT as a salvage modality after definitive radiotherapy. After reviewing elev-

Table 4. Dosimetric parameters of the salvage brachytherapy implants

\begin{tabular}{lccc} 
& 1. Implant & 2. Implant & 3. Implant \\
\hline Prostate D90 (Gy) & 8.33 & 9.0 & 8.35 \\
\hline Prostate V100 (\%) & 85.6 & 90.1 & 85.0 \\
\hline Boost D90 (Gy) & 10.7 & 13.8 & 9.7 \\
\hline Rectum D2 cm 3 (\%) & 55.7 & 52.5 & 58.7 \\
\hline Bladder D2 cm 3 (\%) & 73.4 & 57.2 & 68.0 \\
\hline Urethra D0.1 $\mathrm{cm}^{3}$ (\%) & 114.7 & 127.3 & 115.6
\end{tabular}

$D_{90}$ - dose delivered to $90 \%$ of the CTV, $V_{100}$-volume of CTV receiving $100 \%$ of the dose, $D_{2 \mathrm{~cm}^{3}}$ - maximal dose applied to a volume of $2 \mathrm{~cm}^{3}, D_{0.1 \mathrm{~cm}^{3}}$ - maximal dose applied to a volume of $2 \mathrm{~cm}^{3}$ 
en published series including 424 patients treated with HDR-BT, they concluded that with a 5-year biochemical control ranging from $18-77 \%$, and late grade 3 toxicity between $0-32 \%$ (genitourinary) and $0-5.1 \%$ (gastrointestinal), it represents a safe and efficacious radio-oncological modality. Due to the high inhomogeneity between the different series, including patient characteristics, previous treatments, and different total doses/fractionations, it is difficult to recommend a dose-fractionation schedule or define dose constraints. On the other hand, the oncological results with the different HDR-BT hypofractionated treatments seems to be consistent and reproducible. Even in well selected patients, a second salvage implant seems to be feasible $[9,10]$.

As in the primary treatment setting, limiting the rectal dose represents a challenge for the entire brachytherapy team. Considering that the additional dose applied during the salvage treatment could significantly increase the risk of late toxicity, every effort to reduce the rectal exposure is of paramount importance. In this context, a prospective phase II trial [11] found a rate of rectal fistula as high as $12 \%$. Focal brachytherapy [12] with dose limited only to PET (+) regions could be an interesting approach, especially considering the good anatomical correlation when using ${ }^{68} \mathrm{Ga}$ PSMA as nuclide [13]. But considering the risk of missing subclinical disease, even when using multiparametric MRI [14], it emphasizes the importance to explore these concepts on prospective controlled trials. In this case, we decided to use the information derived from the ${ }^{68} \mathrm{Ga}$ - PSMA PET/CT to define a simultaneous built-in boost region. After discussing with the patient these findings of the PET/CT, he refused a re-biopsy as recommended to histologically prove the residual disease and better define the involved regions. The lack of this information is for sure a limitation of our report. Also, the lack of MRI information during the initial staging and prior to salvage therapy is a further limitation. After a critical review of the initial plans, we could not find any evidence of an underdosage of the PSMA PET $(+)$ region, but the short time interval between initial treatment and PSA rise opts more for a residual disease than a typical relapse, and this represents also a limitation of this report.

A promising approach to limit the rectal dose consists of injecting a biodegradable hydrogel in the virtual plane between the rectum and prostate to increase the distance between both organs and in consequence, reduce the rectal exposure. This is especially effective in brachytherapy treatments due to its characteristic steep dose gradients. Mahal et al. [15] published a series of salvage low-doserate (LDR) BT in eleven previously irradiated patients, including 5 patients with previous BT. In 3 of 11 patients, a gel implant was not feasible (1 patient with prior LDR-BT and 2 patients with prior EBRT). Among the 8 patients with successful injection of the hydrogel (4 prior EBRT, 4 prior LDR-BT), the median space achieved was $10.9 \mathrm{~mm}$ (EBRT) and $7.7 \mathrm{~mm}$ (LDR-BT), with a $\mathrm{p}$ value of 0.048 . Two patients experienced late grade 3-4 toxicity, including a rectal fistula in one patient. The median time to toxicity was 9.9 months and the actuarial estimate of late grade $3-4$ toxicity was $26 \%$ at 16 months. This series shows that in patients treated with salvage LDR-BT, a hydrogel implant after previous radiotherapy is feasible in most cases, but the risk of late toxicity still remains high. Kollmeier et al. [16] retrospectively compared the results of salvage BT after definitive radiotherapy using LDR or HDR BT. They showed a higher peak of urinary symptoms in LDR, but most of the patients returned after 3 years to the baseline.

Recently, van Wijk et al. [17] published a cost-effectiveness analysis using a virtual spacer prior to the placement of an implantable rectum spacer. Using computed tomography scans prior and after the implant of a spacer in 23 patients planned for EBRT (8 planned for hydrogel injection and 15 planned for a rectal balloon implant), they created a virtual spacer and calculated the normal tissue complication probability (NTCP) with and without implant. Then, after the implant, the actual NTCP was calculated. The virtual spacer simulation showed a good correlation with the actual implant, and combined with a cost-effectiveness analysis could help decide which patients will benefit at most of a spacer implantation. Similarly, this could be also an interesting approach in the salvage setting [18].

Since the treatment of this patient, we have injected a spacer in three more patients prior to a salvage HDR-BT with a very favorable acute toxicity profile (only grade 1-2 urinary toxicity). Being more confident with the injection under these conditions, we are planning to start a prospective evaluation of the use of a hydrogel rectal spacer in patients, which are candidates for a salvage HDR-BT after EBRT or HDR-BT initial treatment.

\section{Conclusions}

The use of a hydrogel rectal spacer prior to salvage HDR-BT in a patient previously treated with HDR-BT is feasible. The acute toxicity and good results published so far encouraged us to start a prospective evaluation of this approach.

\section{Disclosure}

Th authors report no conflict of interest.

\section{References}

1. Pinkawa M, Djukic V, Holy R et al. Application of a spacer between prostate and anterior rectal wall to optimize radiotherapy of localized prostate cancer. Strahlenther Onkol 2011; 187 (Suppl): 42-43.

2. Pinkawa M, Berneking V, König L et al. Hydrogel injection reduces rectal toxicity after radiotherapy for localized prostate cancer. Strahlenther Onkol 2017; 193: 22-28.

3. Galalae RM, Zakikhany NH, Geiger F et al. The 15 -year outcomes of high-dose-rate brachytherapy for radical dose escalation in patients with prostate cancer - a benchmark for high-tech external beam radiotherapy alone? Brachytherapy 2014; 13: 117-122.

4. NCCN Prostate cancer version 2.2017. NCCN Clinical Practice guidelines in Oncology. www.nccn.org/professionals/ physician_gls/pdf/prostate.pdf. Accessed October 2017.

5. van den Bos W, Muller BG, de Bruin DM et al. Salvage ablative therapy in prostate cancer: international multidisciplinary consensus on trial design. Urol Oncol 2015; 33: 495.e 1-7.

6. Chade DC, Eastham J, Graefen M et al. Cancer control and functional outcomes of salvage radical prostatectomy for ra- 
diation-recurrent prostate cancer: a systematic review of the literature. Eur Urol 2012; 61: 961-971.

7. Spiess PE, Levy DA, Pisters LL et al. Outcomes of salvage prostate cryotherapy stratified by pre-treatment PSA: update from the COLD registry. World J Urol 2013; 31: 1321-1325.

8. Chatzikonstantinou G, Zamboglou N, Rödel C et al. Highdose-rate brachytherapy as salvage modality for locally recurrent prostate cancer after definitive radiotherapy. A systematic review. Strahlenther Onkol 2017; 193: 683-691.

9. Claren A, Gautier M, Feuillade J et al. Second salvage treatment for local recurrence of prostate cancer using high-doserate brachytherapy: a case report. J Contemp Brachytherapy 2015; 7: 244-247.

10. Maenhout M, van Vulpen M, Moerland M et al. Second salvage high-dose-rate brachytherapy for radiorecurrent prostate cancer. J Contemp Brachytherapy 2017; 9: 161-166.

11. Nguyen PL, Chen MH, D'Amico AV et al. Magnetic resonance image-guided salvage brachytherapy after radiation in select men who initially presented with favorable-risk prostate cancer: a prospective phase 2 study. Cancer 2007; 110: 1485-1492.

12. Guimas V, Quivrin M, Bertaut A et al. Focal or whole-gland salvage prostate brachytherapy with iodine seeds with or without a rectal spacer for postradiotherapy local failure: How best to spare the rectum? Brachytherapy 2016; 15: 406-411.

13. Zamboglou C, Schiller F, Fechter T et al. (68) Ga-HBED-CC PSMA PET/CT Versus Histopathology in Primary Localized Prostate Cancer: A Voxel-Wise Comparison. Theranostics 2016; 6: 1619-1628.

14. Peters M, Maenhout M, van der Voort van Zyp JR et al. Focal salvage iodine-125 brachytherapy for prostate cancer recurrences after primary radiotherapy: a retrospective study regarding toxicity, biochemical outcome and quality of life. Radiother Oncol 2014; 112: 77-82.

15. Mahal BA, Ziehr DR, Hyatt AS et al. Use of a rectal spacer with low-dose-rate brachytherapy for treatment of prostate cancer in previously irradiated patients: Initial experience and short-term results. Brachytherapy 2014; 13: 442-449.

16. Kollmeier MA, McBride S, Taggar A et al. Salvage brachytherapy for recurrent prostate cancer after definitive radiation therapy: A comparison of low-dose-rate and high-doserate brachytherapy and the importance of prostate-specific antigen doubling time. Brachytherapy 2017; 16: 1091-1098.

17. van Wijk Y, Vanneste BGL, Walsh S et al. Development of a virtual spacer to support the decision for the placement of an implantable rectum spacer for prostate cancer radiotherapy: Comparison of dose, toxicity and cost-effectiveness. Radiother Oncol 2017; 125: 107-112.

18. Vanneste BG, Pijls-Johannesma M, van De Voorde L et al. Spacers in radiotherapy treatment of prostate cancer: is reduction of toxicity cost-effective? Radiother Oncol 2015; 114: 276-281. 